\title{
Comparison Of Periodontal Health Of Orthodontic And Non-Orthodontic Patients
}

\author{
Natasha Zaidi, Yousuf Moosa, Syed Ahmed Omer, Saima Mazhar, Kulsoom Fatima Rizvi, Amir Akbar Shaikh
}

Objective: To compare the periodontal health status among orthodontic and non-orthodontic groups aged between 14-30 years of both genders.

Study design And Setting: This comparative cross sectional study was conducted at Department of Orthodontics and Periodontics from January 2019-till June 2019 at dental Hospital of Bahria Dental College Karachi.

Methodology: Those patients aged 14-30 under orthodontics treatment for about 3 months and were able to give consent were included in the study. Periodontal status among both groups (65 orthodontics and 65 non orthodontic) patients was evaluated with the help of CPITN probe on index teeth. To assess the oral hygiene status; the questionnaire was formulated regarding the use and frequency of toothbrush; use of interdental cleaners such as interdental brush, use of mouthwash, use of tongue cleaners and dental appointments. A single observer from periodontics department was appointed for observation and record data of patients coming to orthodontic department and to carry out a clinical examination. The data was scrutinized using SPSS 23, and using the chi-square test various other comparisons were performed.

Results: Statistically significant association was observed in CPITN scores between the orthodontic and non-orthodontic patients $(\mathrm{p}<0.05)$. Patients who underwent orthodontic treatment had increased plaque accumulation and probing depth which resulted in periodontal tissue destruction.

Conclusion: It was concluded that the periodontal condition of ortho patients was deteriorating as compare to non ortho patient. There was no noticeable attachment loss found that could lead to mobility of the tooth and its loss.

Key words: calculus, non-orthodontics, orthodontics, periodontal status, plaque.

\section{INTRODUCTION:}

Orthodontic treatment has extensive effect on dentofacial complex. It helps in proper alignment of dentition by orthodontic treatment modalities; improving occlusion, enhancing esthetics and maintaining oral hygiene. Oral hygiene can be affected by different abnormalities in teeth alignment. Plaque retention is one of the causative factor leads to imbalance in micro biota of oral cavity; further accumulation of plaque results in malocclusion which is more often seen in crowded dentition; commonly a feature

I Natasha Zaidi

Senior Lecturer, Department of Periodontology

Bahria University Medical and Dental College

I Email: natashazaidi85@gmail.com

I Yousuf Moosa

I Associate Professor \& HOD Periodontology

I Sir Syed College of Medical Sciences for Girls

I Syed Ahmed Omer

I Assistant Professor \& HOD Department Science of Dental

Material, Bahria University Medical and Dental College, I Karachi

I Saima Mazhar

I Senior Lecturer, Department of Periodontology

I Bahria University Medical and Dental College

I Kulsoom Fatima Rizvi

Associate Professor \& HOD Community Dentistry

Bahria University Medical and Dental College

Amir Akbar Shaikh

Associate Professor, Department of Community Dentistry

I Sir Syed College of Medical Sciences for Girls

Received: 12-Jul- 2019

I Accepted: 17-Feb-2020

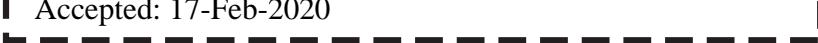

of lower anterior. Open bite, spacing, cross bite impacted teeth, crowding, narrow arches and rotation of teeth are the atypical features of malocclusion. ${ }^{1}$

The initial inflammation of gingival tissue occurring around the gums, surrounding the teeth is known as gingivitis, when left untreated progresses into periodontal disease. On the other hand; the fixed orthodontic appliances; when horn in the gingival sulcus rendered plaque accumulation; results in food deposition. This cementation of ortho appliance and the food accumulation results while brushing and at times it is difficult to clean that debris further deteriorating the oral health. ${ }^{2}$ Since patient fails to maintain appropriate oral hygiene, they are likely to develop gingivitis as it progresses within 21 days of its occurrence. ${ }^{1}$

Fixed orthodontic appliances increases the growth of microbial flora due to deposition of plaque and calculus and results in bleeding gums. ${ }^{3-7}$ and other periodontal associated problems such as gingival recession, gingival hypertrophy, alveolar bone hammering, pocketing. ${ }^{8,9}$ The predominant initiators of plaque are gram positive organisms which later get replaced by gram negative and then the anaerobes. ${ }^{1}$

Oral hygiene preservation during orthodontic treatment is only possible by proper teaching to the patient, their enthusiasm, collaboration and their approach towards their treatment played an active role to maintain oral hygiene..$^{10} \mathrm{~A}$ lot of researches proved that patients either lack knowledge of proper hygiene maintenance or show carelessness in maintaining it. Despite having the knowledge some patients still fail to follow proper oral hygiene instruction. ${ }^{11,12}$ 
In order to overcome caries and periodontal problems during orthodontic treatment; there is a need to educate patients regarding maintenance of oral hygiene and how to control the calculus deposition ${ }^{13}$ and indeed it was the rationale of the study to assess the periodontal health among orthodontic patients and further to educate them accordingly. It is important to guide orthodontic patients about oral hygiene maintenance before starting the treatment. ${ }^{14}$ To address this; it is essential to practice proper brushing technique regularly. Awareness regarding different types of orthodontic appliances and brushes should be provided to the patients especially the interdental brushes. ${ }^{15}$ Therefore this study was aimed to compare the periodontal health status of orthodontic and non orthodontic patients.

\section{METHODOLOGY:}

All those patients who were aged between 14-30 years of age and visited to orthodontic and periodontics department for regular dental check-up in the Dental Hospital of BUMDC from January 2019 to June 2019 were examined for this study. This study was conducted after the approval obtained from Ethical Review Committee of BUMDC. Those patients aged 14-30 under orthodontics treatment for about 3 months and were able to give consent were included in the study; while those patients having pathological or congenital condition such as craniofacial anomalies because of the compromised periodontal tissue condition and those who were seeking prosthesis treatment specially removable prosthetic appliances were excluded from the study. The Sample size was calculated by considering the prevelance of $10 \%{ }^{1}$. The calculated sample size was 139 subjects .Total 65 patients from each respective department were taken into consideration were divided into test group (orthodontic patients) and control groups (non-orthodontic patients). Gender distribution details filled in figure 1. A single observer from periodontics department was appointed for observation and record data of patients coming to orthodontic department and to carry out a clinical examination. To obtain intraexaminer reliability, ten patients were examined on two occasions at a 1-week interval using the Community Periodontal Index and kappa test value of 0.95 was obtained.

Periodontal status among both groups was evaluated with the help of CPITN probe on index teeth. ${ }^{2}$ To assess the oral hygiene status; the questionnaire was formulated and included questionnaire were regarding the use and frequency of toothbrush; use of interdental cleaners such as interdental brush, use of mouthwash, use of tongue cleaners and dental appointments.

The periodontal condition of all the patients was assessed conferring to WHO protocol, by the use of an axenic CPITN probe on the index teeth and a mouth mirror under a favorable light source. A tooth was examined to obtain the pocket depth, spot calculus and to check any bleeding response. The areas chosen for examination were the distal, midline and mesial aspects of the facial and palatal/lingual areas. The measure of the depth of gingival sulcus or pocket was carefully examined with the tip of the probe. The probing depth was measured by introducing the probe alongside the long axis of the tooth, between the tooth and gingiva starting at the distobuccal area of the index tooth. The corresponding maximum score for each sextant was calculated and noted down in the appropriate box. The dentition was classified into 6 sextants. The CPITN code noted on the index teeth according to their status were $0,1,2,3,4$, denoting healthy gums, bleeding gums on the use of probe, presence of calculus on the gingival surfaces, existence of a low pocket of about 4 to $6 \mathrm{~mm}$ or a profound pocket measuring about $6 \mathrm{~mm}$ or more. While $\mathrm{x}$ decoded a sextant with less than 2 teeth (excluded sextant).

The collected data was analyzed using the Statistical Package for Social Sciences (SPSS) version 23 and comparisons of CPITN codes were performed using the chi-square test, with a value of $\mathrm{P}<0.05$ regarded as significant.

\section{RESULTS:}

There were total 130 patients evaluated and comprised of 35 males and 95 females within 14-30 years of age. The periodontal pockets score was compared among both groups. It was revealed CPITN score was $57 \%$ in ortho group having periodontal pockets scoring of 3 and 4 on CPITN probe; and $(17 \%)$ in non-orthodontic group pocket depth with same score ( 3 and 4 score) on CPTIN probe. The elevation in periodontal disease was found to be statistically significant between ortho and non-ortho groups $(\mathrm{p}<0.05)$ (Table-1).

A CPITN score of 3 and 4 indicated the presence of a pocket in $4(6 \%)$ patients of orthodontic group who were interdental brushers. At the same time the same CPITN score of 3 and 4 was also found in about $34(52 \%)$ patients who were not using the interdental brushes-Graph-2.

According to the results; from the total 130 patients, 4 of 40 interdental brush users that was about $10 \%$ had 3 and 4 CPITN score along with the periodontal pockets. Periodontal pockets were present in $46(51 \%)$ of the 90 patients who were not using any sort of interdental assistance and CPTIN score was 3 and 4. (Graph-3). Table 3-depicted the progression of periodontal disease $(\mathrm{p}<0.05)$ among patients who are not using dental aids to the ones using it.

According to the responses revealed: both groups were brushing their teeth with a tooth brush ranging $66 \%$ of them doing it once every day while $30 \%$ did it twice daily and only $1 \%$ doing it thrice daily. Whilst when it came to the use of interdental cleaners, in the ortho group $47 \%$ were interdental brush users, and in the non-ortho group, only $15 \%$ patients used dental floss. Volunteered tongue cleaners were $12 \%$ from orthodontic group and 5\% from nonorthodontic. At the same time use of mouth wash was practiced by $10 \%$ orthodontic patients and 38\% normal patients not undergoing ortho treatment. Total $20 \%$ patients 
of orthodontic follow up and $10 \%$ were routine diagnostic patients.

Figure 1: Gender Distribution among Ortho and Non-Ortho patients

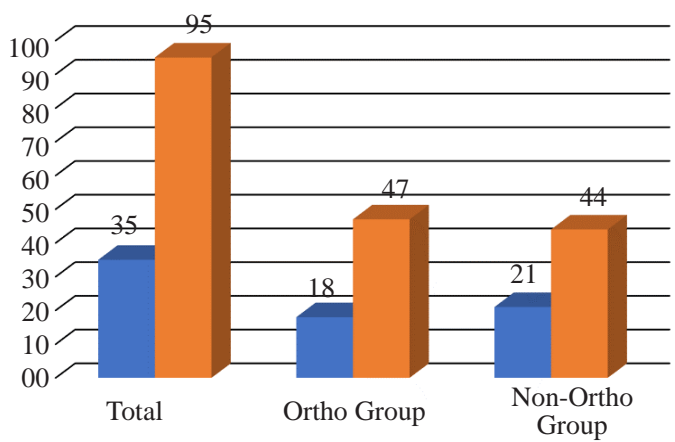

Figure- 2. Comparing the CPITN scores among Interdental and non-Interdental brush users among Orthodontic patients

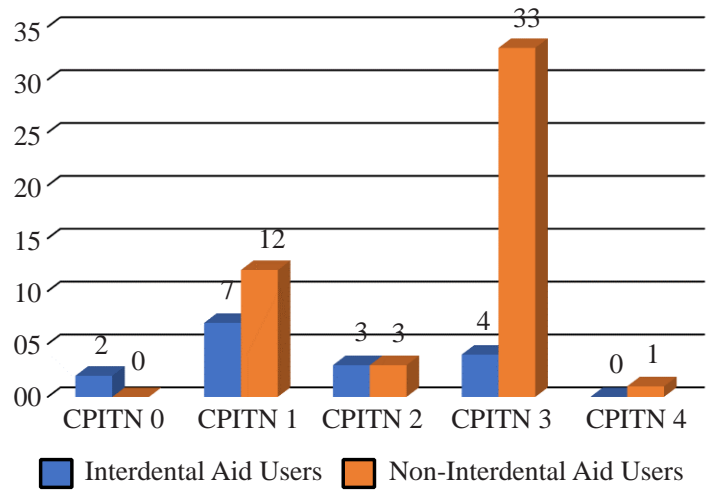

Figure 3: Relationship of CPITN Scores between Interdental and non-interdental Brush users

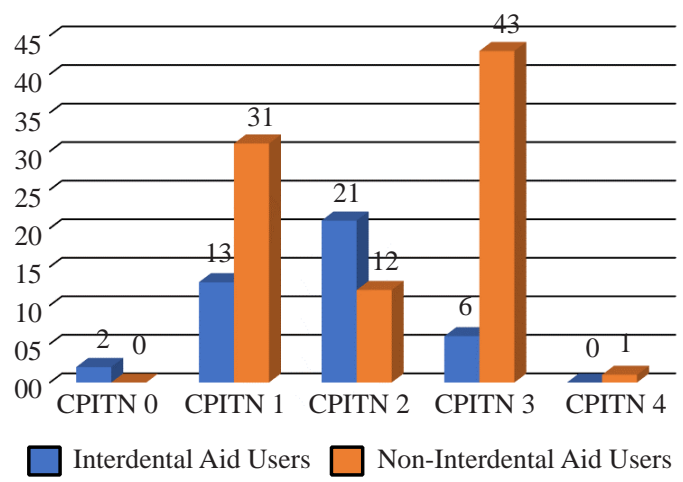

Table 1: Comparison of CPITN Score among Ortho and NonOrtho groups

\begin{tabular}{|c|c|c|c|}
\hline $\begin{array}{c}\text { CPITN } \\
\text { Score }\end{array}$ & $\begin{array}{c}\text { Ortho group } \\
\text { N=65 }\end{array}$ & $\begin{array}{c}\text { Non-Ortho } \\
\text { group N=65 }\end{array}$ & P-Value \\
\hline 0 & 2 & 13 & \multirow{2}{*}{$0.000^{*}$} \\
\hline 1 & 20 & 31 & \\
\hline 2 & 6 & 10 & \\
\hline 3 & 36 & 11 & \\
\hline 4 & 1 & 0 & \\
\hline
\end{tabular}

Table 2: Comparison of CPITN Score between interdental and non-interdental aid users among all patients

\begin{tabular}{|l|c|c|c|}
\hline \multicolumn{1}{|c|}{ CPITN } & Score 0,1,2 & Score 3,4 & p-value \\
\hline $\begin{array}{l}\text { Interdental aid users } \\
(\mathrm{N}=40)\end{array}$ & $36(90 \%)$ & $4(10 \%)$ & \\
\cline { 1 - 3 } $\begin{array}{l}\text { Non-interdental aid users } \\
(\mathrm{N}=90)\end{array}$ & $44(48 \%)$ & $46(51 \%)$ & 0.000 \\
\hline
\end{tabular}

\section{DISCUSSION:}

The purpose of the study was to compare the periodontal health among patients seeking orthodontic help to the ones not undergoing orthodontic care. In this study; the CPITN score was increased among orthodontic treatment receiving patients. Momina et al. ${ }^{16}$ revealed that the arrangement of brackets provides retentive sites and bionomic surroundings for accumulation of biofilm, furthermore the CPITN scores observed in this study harmonize with those fallouts. Excessive bleeding and inflammation worsen the periodontal health resulting in surveillance of noticeable raise in plaque and gingival index of test group. Three months after placement of fixed appliances there was foremost augment in clinical and microbial characteristics, comparable outcomes shown by Marda et al. ${ }^{17}$

Undoubtedly, the CPITN score of orthodontic and non orthodontic groups were contradicting, admitting the fact that score for group using interdental brush were lower as compared to the ones not using brushing. Since orthodontic appliances placement hinder maintenance of proper oral hygiene causing deposition of plaque leading to increase in pocket depth, which directly contributes to increase in CPITN score and the same observation reported by Nasir et al. ${ }^{18}$

As reported by Angelina et al, ${ }^{19}$ the most affected area periodontally in orthodontic patient is the interdental area which agrees with the observation of this study. This truly confirms that orthodontic appliances are prone to plaque accumulation, eventually initiating periodontal problems. These results were in agreement with other studies. ${ }^{20} \mathrm{~A}$ clinically healthy gingiva has ascertained depth which depicted by different methods as $1.5 \mathrm{~mm}$ and $1.8 \mathrm{~mm}^{21}$

Radiographic analysis indicated the areas of bone loss presuming the formation of pockets. ${ }^{22}$ This study agrees with Fatemah et $\mathrm{al}^{23}$ who reported orthodontic patients bearing fixed appliances have slight attachment loss with increase in pocket depth.

The primary etiological and commencing factor for any gingival or periodontal regression is plaque and its cleanliness becomes a challenge by fixed appliances placement. Earlier colonies initiating plaque are gram positive rods and cocci, transforming into gram negative and anaerobic organisms introducing periodontal defects. ${ }^{24}$ Orthodontic treatments render the patient to achieve appropriate oral hygiene during the treatment period. This in turn causes plaque accumulation 
which can trigger pathological conditions, since orthodontic appliances serve as mechanical trappers for plaque. Henceforth periodontal conditions in orthodontic patients can be bypassed by enduring plaque control therapy.

Encouraging the adolescents into proper oral hygiene maintenance can reduce the burden of dental distress. ${ }^{25,26}$ Oral hygiene tools for orthodontic patients such as floss threaders, interdental brushes are available to control the plaque accumulation. $^{27}$

The limitation of the study was the effects of orthodontic treatment upon periodontal health. Further studies can be conducted to assess the periodontal health after the exclusion of orthodontic appliances. Therefore; in a nutshell, proper oral hygiene measures along with interdental brushes can reduce the plaque accumulation.

\section{CONCLUSION:}

It was concluded that the periodontal condition of ortho patients was deteriorating as compare to non ortho patient There was no noticeable attachment loss found that could lead to mobility of the tooth and its loss.

\section{REFERENCES:}

1. Moosa Y, Han LN, Safdar J, Sheikh OA, Pan YP. Periodontal status of Pakistani orthodontic patients. Brazilian Oral Research. 2015; 29(1): 1-5.

2. Leonardo T, Roberto F, Cleverson OS, Dimitris NT. Plaqueinduced gingivitis: Case definition and diagnostic considerations. J Periodontol. 2018; 89(Suppl 1):S46-S73.

3. Irum N, Aamir MB, Uzma B, Hina M. Periodontal tissue; effect of fix orthodontic treatment. Professional Med J. 2017; 24(6):930-934.

4. Amrinder ST, Nitin B. Gingival Hyperplasia a Sequalae of Orthodontic Therapy- A Case Report. Acta Scientific Dental Sciences. 2018; 2(6): 84-86.

5. Hala A, Nayer A, Khaled AEG. Management of Gingival Hyperplasia in Patients with Fixed Orthodontic Appliances Using Topical Herbal Preparations (A Comparative Clinical and Microbiological Study. Acta Scientific Dental Sciences. 2019; 3(1): 02-11.

6. Alice SP, Luana SA, Julio E, Fabrýcio BZ, Marisa M. Gingival enlargement in orthodonticpatients: Effect of treatment duration. American Journal of Orthodontics and Dentofacial Orthopedics. 2017; 152(4): 477-482.

7. Silvia Ci, Guido P, Roberta C, Loredana C. Orthodontic Fixed Appliance and Periodontal Status: An Updated Systematic Review. Open Dent J. 2018; 12: 614-622.

8. Bollen AM, Cunha-Cruz J, Bakko DW, Huang GJ, Hujoel PP. The effects of orthodontic therapy on periodontal health: a systematic review of controlled evidence. The Journal of the American Dental Association. 2008 Apr 30; 139(4):41322.

9. Ristic M, Vlahovic Svabic M, Sasic M, Zelic O. Clinical and microbiological effects of fixed orthodontic appliances on periodontal tissues in adolscents. Orthod Craniofac Res 2007; 10:187-195.

10. Kadu A, Chopra SS, Gupta N, Jayan B, Kochar GD. Effect of the personality of the patient on pain perception and attitude towards orthodontic treatment. J Indian Orthod Soc. 2015;49(2):89-95.
11. Elanchezhiyan S, Raja. Awareness on gingival health among orthodontic correction seeking individuals. J Indian Acad Dent Spec Res. 2010;1(3):19-21.

12. Anuwongnukroh N, Dechkunakorn S, Kanpiputana R. Oral Hygiene Behavior during Fixed Orthodontic Treatment. Dentistry. 2017; 7(10): 1-5.

13. Baheti MJ, Toshniwal NG. Survey on oral hygiene protocols among orthodontic correction-seeking individuals. J Edu Ethics Dent.2015;5(1):8-13.

14. Arici S, Alkan A, Arici N. Comparison of different toothbrushing protocols in poor-tooth brushing orthodontic patients. Eur J Orthod. 2007;29(5):488-92.

15. Matiæ S, Ivanoviæ M, Nikoliæ P. Evaluation of a prevention programme efficiency for patients with fixed orthodontic appliances. Vojnosanit Pregl. 2011;68(3):214-9.

16. Momina T, Anam A, Muhammad MS. Study to know outcome of orthodontic treatment and its effect on periodontal health. Indo Am. J. P. Sci. 2018; 05(08): 8249-8253.

17. Marda A,Elhamzaoui S, MansariAE, Souly K, Farissi F, Zouhdi M, Zaoui F, Bahije L. International Journal of Dentistry. 2018; article ID 5939015. Published online 2018. Doi.org/10. $1155 / 2018 / 5939015$.

18. Nasir N, Ali S, Bashir U, Ullah A. Effect of orthodontic treatment on periodontal health. Pakistan Oral \&Dental Journal. 2011; 31(1): 111-114..

19. Angelina G, Giorgio P, Anna B, Giampietro F, Giulio R. International Journal of Dentistry. 2016; article ID 4723589. Published online 2016. Doi.org/10.1155/2016/4723589.

20. Thilagrani PR, Abhay PPA, Muqtadir SMQ, Hemlata R, Anurag T, Debashish D. Association of Periodontal Health with Orthodontic Appliances among Indian Patients. J Int Oral Health. 2015; 7(1): 44-47.

21. Vandana KL, Shivani S, Savitha B, Vivek HP. Assessment of Gingival Sulcus Depth, Width of Attached Gingiva, and Gingival Thickness in Primary, Mixed, and Permanent Dentition. Journal of Dental Research and Review. 2017; 4(2):42-49.

22. Elham SAA, Eman MA, Dafi QT. Periodontal health knowledge and awareness among subjects with fixed orthodontic appliance. Dental Press Journal of Orthodontics. 2018; 23(5):40.e1-40.e9.

23. Fatemah AA, Faiza AO. The Effect of Fixed Orthodontic Appliances Versus Clear Aligner on Periodontal Health: A Review of the Literature. Journal of Dental and Medical Sciences. 2018; 17(1): 19-22.

24. Spyridon NP, Anastasia AP, Theodore E. Effect of orthodontic treatment on periodontal clinical attachment: A systematic review and meta-analysis. European Journal of Orthodontics. 2017. Published online 2017. DOI: 10.1093/ejo/cjx052.

25. Amal HA, Lara TE. Oral Hygiene Performance among a Sample of Sudanese Orthodontic Patients. Journal of Dentistry and Oral Hygiene. 2015; 1(1): 1-8.

26. Huang J, Yao Y, Jiang J, Li C. Effects of motivational methods on oral hygiene of orthodontic patients. A systematic review and meta-analysis. 2018; 97(47): 1-8.

27. Lu H, Tang H, Zhou T, Kang N. Assessment of the periodontal health status in patients undergoing orthodontic treatment with fixed appliances and invisalign system: A meta-analysis. Medicine. 2018; 97(13):1-10.

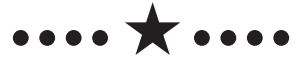

